\title{
Dendritic mechanisms controlling spike-timing-dependent synaptic plasticity
}

\author{
Björn M. Kampa ${ }^{1}$, Johannes J. Letzkus ${ }^{2}$ and Greg J. Stuart ${ }^{2}$ \\ ${ }^{1}$ Brain Research Institute, University of Zürich, Winterthurerstr. 190, 8057 Zürich, Switzerland \\ ${ }^{2}$ The John Curtin School of Medical Research, Australian National University, Mills Rd, ACT 0200, Canberra, Australia
}

\begin{abstract}
The ability of neurons to modulate the strength of their synaptic connections has been shown to depend on the relative timing of pre- and postsynaptic action potentials. This form of synaptic plasticity, called spike-timingdependent plasticity (STDP), has become an attractive model for learning at the single-cell level. Yet, despite its popularity in experimental and theoretical neuroscience, the influence of dendritic mechanisms in the induction of STDP has been largely overlooked. Several recent studies have investigated how active dendritic properties and synapse location within the dendritic tree influence STDP. These studies suggest the existence of learning rules that depend on firing mode and subcellular input location, adding unanticipated complexity to STDP. Here, we propose a new look at STDP that is focused on processing at the postsynaptic site in the dendrites, rather than on spike-timing at the cell body.
\end{abstract}

\section{Introduction}

Adaptation and learning are among the most remarkable abilities of the brain. Neurons modify their synaptic connections to adapt to changes in sensory input, for example after sensory deprivation caused by the surgical closure of the eyes [1]. Changes in synaptic strength are also thought to underlie learning and storing of new memories. Experimental evidence for long-term changes in synaptic strength was first shown in the hippocampus of rabbits, where tetanizing perforant path afferents to the dentate gyrus induced long-term potentiation (LTP) of these synaptic connections [2]. Later, changes in the strength of synaptic connections leading to long-term depression (LTD) were also described [3].

The characteristics of the induction of LTP or LTD resembled a model for memory storage previously suggested by Donald Hebb. This model was based on the idea that neurons that are active at the same time strengthen their synaptic connections ('fire together wire together') [4] (Figure 1a). The molecular candidate detecting synchronous firing in pre- and postsynaptic neurons was soon found to be the NMDA receptor, which functions as a coincidence detector. It opens its $\mathrm{Ca}^{2+}$-permeable pore only when presynaptic glutamate release is coincident with postsynaptic depolarization, leading to removal of the voltage-dependent

Corresponding author: Kampa, B.M. (kampa@hifo.unizh.ch).

Available online 31 August 2007. magnesium block inside the NMDA channel pore [5,6]. Given that many forms of LTP and LTD are dependent on NMDA receptor activation, bi-directional changes in Hebbian synaptic plasticity are thought to result as strong activation of the NMDA receptor leads to a large $\mathrm{Ca}^{2+}$ influx at the postsynaptic site that induces LTP, whereas moderate NMDA receptor activation and $\mathrm{Ca}^{2+}$ influx is thought to cause LTD [7].

\section{The classical STDP model}

In recent years, a variation of this form of synaptic plasticity has been described that involves pairings of only a single pre- and postsynaptic action potential, in which the sign and magnitude of the change in synaptic strength are determined by the relative timing of spikes in the connected neurons (Figure 1b). This form of synaptic plasticity has been named spike-timing-dependent plasticity (STDP) $[8,9]$. Whereas in many cases STDP has been shown to depend on NMDA receptor activation, spike-timing-dependent LTD can also be induced via activation of metabotropic glutamate receptors $[10,11]$ and after dendritic release of endocannabinoids [12,13].

Typically synapses that have transmitted an excitatory postsynaptic potential (EPSP) just before the generation of a postsynaptic action potential will be potentiated (positive timing), whereas EPSPs arriving after action potential generation undergo synaptic depression (negative timing; although see $[11,14,15])$. In other words, synapses that contribute to the depolarization leading to postsynaptic firing will be enhanced, whereas uncorrelated synaptic inputs will be suppressed. Given that most synaptic inputs are made onto dendrites, signalling to the synapse that the neuron has generated an output is thought to be mediated by backpropagating action potentials [16]. Action potentials, once generated in the axon $[17,18]$, not only propagate along the axon towards presynaptic terminals, but are also capable of propagating backwards into the dendritic tree in many neurons [19]. There, they provide the necessary depolarization to relieve the $\mathrm{Mg}^{2+}$ block of the NMDA receptors [20,21], which is essential for STDP induction $[9,22]$. The timing of backpropagating action potentials relative to glutamate binding to NMDA receptors determines the amount of NMDA receptor activation $[15,20]$ and hence $\mathrm{Ca}^{2+}$ influx [11,23,24]. Different levels of postsynaptic $\mathrm{Ca}^{2+}$ are thought to activate different signalling 


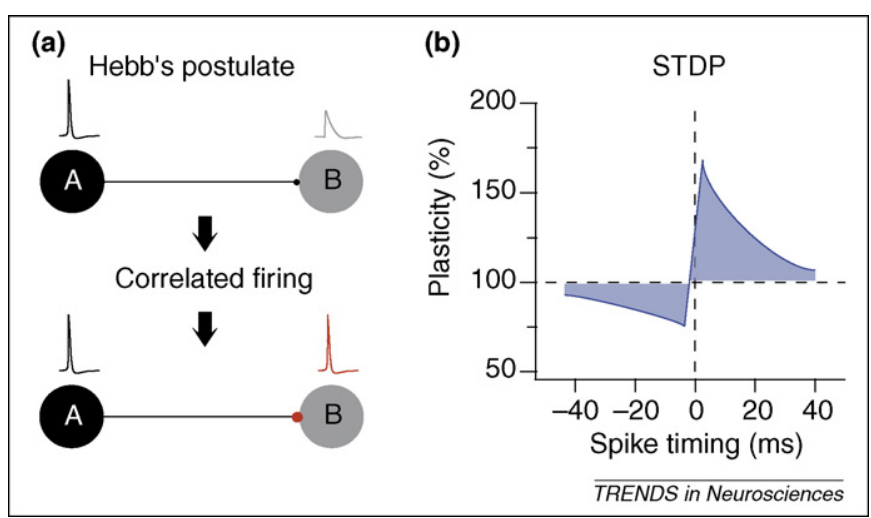

Figure 1. Classical view of STDP. (a) Schematic representation of Hebb's postulate (1949) [4], which states that if neuron A repeatedly participates in firing cell $B$, the efficiency of cell $A$ as one of the neurons firing cell $B$ is increased. (b) Classical STDP timing curve providing experimental support for Hebb's postulate by defining the time window in which correlated firing leads to LTP. In addition, these data show that negative timings (uncorrelated activity) cause depression. Positive spike timing refers to when the presynaptic action potential precedes the postsynaptic action potential and negative spike timing the opposite situation. Adapted with permission from [9].

cascades, thereby controlling the sign and magnitude of the induced synaptic change [25].

New research indicates that the postsynaptic depolarization necessary for STDP induction is greatly influenced by active dendritic mechanisms, as well as the dendritic location of synapses. In this review we summarize recently published studies on the role of dendrites in STDP induction and provide new insights into Hebbian (and non-Hebbian) learning rules for STDP induction in a physiological context.

\section{Beyond classical STDP}

The classical STDP model refers to somatic action potential timing as a reference for the spike-timing window [9]. In most neurons, however, the vast majority of synapses are located on dendrites at sites remote from the soma and the site of action potential initiation in the axon. In layer 5 pyramidal neurons, for example, most synapses are located at distances about $150 \mu \mathrm{m}$ from the soma, and some synapses occur as far as $1000 \mu \mathrm{m}$ from the soma [26]. Action potential backpropagation into the dendrites of these neurons is decremental [19] and can fail at distal sites in the dendritic tree [27]. This observation might explain why pairing synaptic inputs with backpropagating action potentials at low frequencies $(\leq 1 \mathrm{~Hz})$ can fail to induce LTP in both cortical and hippocampal neurons in acute slice preparations [15,28-31] (Figure 2a,b). Other studies using extracellular stimulation or paired recordings in culture have observed STDP induction during pairing with single action potentials at low frequencies [9,31-33]. This might occur as synaptic inputs in cultured neurons, and during extracellular stimulation, activate more synaptic connections [34] leading to a significantly larger local depolarization at the site of synaptic input, which when combined with backpropagating action potentials can be sufficient for STDP induction [31].

It has been further shown that high-frequency trains $(>10 \mathrm{~Hz})$ of action potentials interleaved with synaptic stimulations reliably induce STDP at cortical synapses $[29,31]$. In addition to the problem that these pre- and postsynaptic action potential trains have ambiguous spike-timing intervals (both positive and negative), cortical neurons have recently been shown to fire action potentials at very low rates in anaesthetized, sleeping and awake behaving animals, even during sensory stimulation [35-37], making it unlikely that the required pattern of correlated pre- and postsynaptic activity would occur in vivo at sufficiently high rates. Is STDP, therefore, unlikely to occur in the brain during natural stimuli? Or is the classical model of STDP too simple? Several recent studies point towards a more complex STDP model incorporating the morphology of neurons and the active properties of their dendrites. In the following sections of this review, we summarize these mechanisms focusing on (i) the role of active dendritic properties such as dendritic voltage-gated $\mathrm{Ca}^{2+}$ and $\mathrm{Na}^{+}$channels and (ii) the influence of synapse location within the dendritic tree on STDP learning rules.

\section{Dendritic mechanisms controlling STDP I: role of dendritic spikes}

Synaptic plasticity is generally induced by coincidence of a synaptic input with postsynaptic depolarization. In classical STDP, appropriately timed postsynaptic action potentials trigger LTP by depolarizing and unblocking glutamatebound NMDA receptors [16]. In cases where backpropagation is decremental or fails completely, STDP can still be induced if action potential backpropagation is rescued by sufficient postsynaptic depolarization. This can be provided by current injection during whole-cell recordings $[28,31,38]$ or by stimulation of action potential bursts $[15,28,30]$ (Figure 2). High-frequency action potential bursts are naturally occurring firing patterns in many pyramidal neurons [39] and can generate dendritic spikes leading to strong depolarization of large parts of the dendritic tree [40-42] (Box 1). It has been shown that only bursts above a critical frequency $(\sim 100 \mathrm{~Hz})$ of action potential firing induce dendritic spikes $[40,41]$ (see Box 1). There is also evidence that action potential bursts need to exceed a critical frequency to induce STDP [15] (Figure 2d), suggesting a requirement of dendritic spikes. Similarly, inhibition of dendritic spikes by voltage-gated $\mathrm{Ca}^{2+}$ channel antagonists can block induction of STDP at synapses on basal [15] and apical [28] dendrites of cortical layer 5 pyramidal neurons (Figure 2d). These results suggest that global dendritic spikes during burst firing are required for induction of LTP during low-frequency pairing. This form of plasticity is Hebbian in that it correlates presynaptic input with postsynaptic action potential firing.

By contrast, non-Hebbian synaptic plasticity can also be induced by dendritic spikes when they occur in isolation from axonal action potentials. The first evidence for this was provided by Golding and co-workers [43], who demonstrated that LTP at distal synapses onto hippocampal CA1 pyramidal neurons can be induced by repetitive, highfrequency stimulation eliciting local dendritic spikes in the absence of axonal action potentials. More recently, it has been shown that pairing of synaptic input with local NMDA spikes in conjunction with application of brain-derived neurotrophic factor (BDNF) is required for induction of LTP at distal inputs onto basal dendrites of pyramidal neurons in somatosensory cortex [44]. It is 
(a)

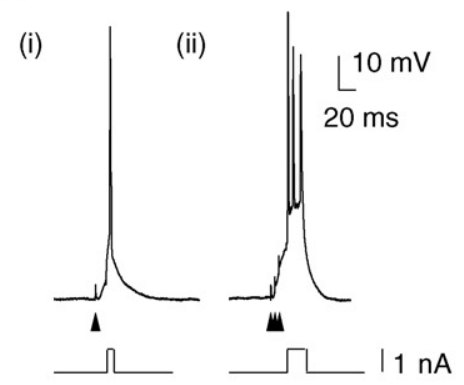

(b)

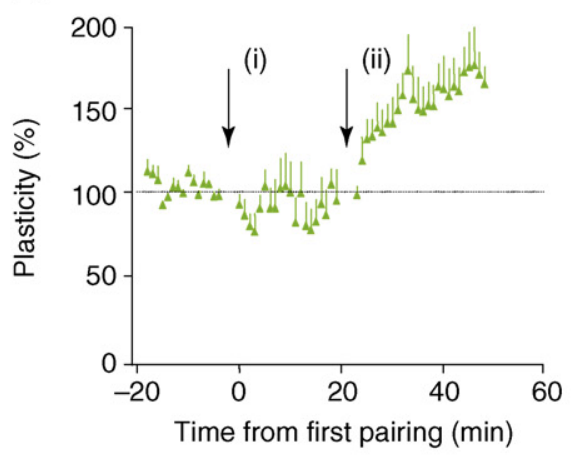

(d)

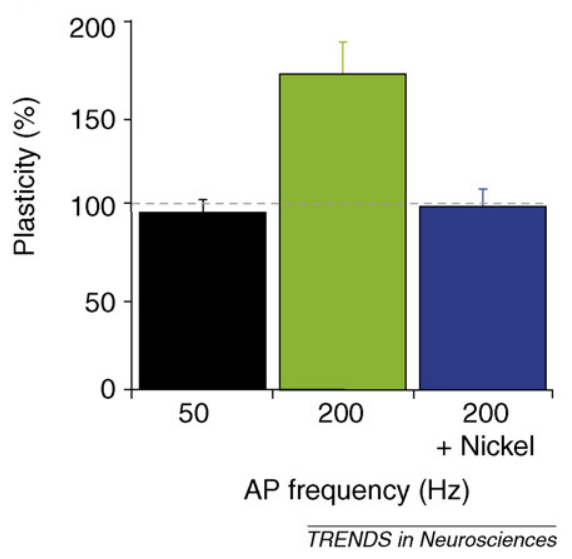

Figure 2. Requirement of dendritic spikes for STDP induction. (a,b) LTP induction in the hippocampus requires postsynaptic burst firing. (a) The two firing patterns (i) and (ii) used for plasticity induction in CA1 pyramidal neurons. Extracellular synaptic stimulation (triangles) was paired with intracellular current injections (bottom traces) to evoke postsynaptic action potentials (top traces) at positive timing intervals (EPSP stimulation before postsynaptic action potential generation). (b) Pairing single EPSPs and action potentials (i) failed to induce LTP, whereas pairing EPSPs with action potential bursts (ii) induced robust LTP. Adapted with permission from [30]. (c,d) LTP induction in layer 5 pyramidal neurons requires generation of high-frequency action potential bursts and dendritic spikes. (c) Plasticity induction paradigm in pairs of layer 5 pyramidal neurons. Unitary EPSPs were evoked by single presynaptic action potentials (top trace) just prior to the onset of three postsynaptic action potentials (positive spike timing) at $50 \mathrm{~Hz}$ or $200 \mathrm{~Hz}$. Bottom trace shows STDP induction with action potential bursts at $200 \mathrm{~Hz}$ in the presence of nickel. The arrow marks reduction in the afterdepolarization, indicating blockade of dendritic spike induction in the presence of nickel (see Box 1). (d) Only pairing with postsynaptic action potentials at $200 \mathrm{~Hz}$, which is above the critical frequency for dendritic spike generation (see Box 1), induces LTP. In addition, LTP was abolished when dendritic spikes were blocked by bath application of nickel. Adapted with permission from [15].

worth noting here that whereas these basal inputs are physically not very far from the soma (100 to $200 \mu \mathrm{m})$ they are electronically distal [45]. By contrast, generation of only a single local dendritic spike induces LTD in layer 5 pyramidal neurons in the visual cortex [46]. What physiological role could this non-Hebbian synaptic plasticity serve? One possibility is that LTP induced by local dendritic spikes will enhance the ability of the potentiated inputs to influence generation of axonal action potentials. Conversely, non-Hebbian LTD could serve to prevent the unbalanced distribution of synaptic weights across the dendritic tree [47]. Interestingly, it has been demonstrated that the forward propagation of distal dendritic spikes can be enhanced by activation of proximal inputs [48], making it possible that non-Hebbian plasticity can be switched to Hebbian STDP depending on the activity level of proximal inputs.

In summary, recent studies indicate that dendritic spikes are required for induction of both Hebbian (STDP) and non-Hebbian plasticity in many cortical neuron types under various experimental regimes [15,28,43,44,46,49]. These findings suggest that local dendritic properties exert a powerful control over the modulation of synaptic inputs

\section{Box 1. Dendritic spikes}

Dendrites of many neuronal types express a wide variety of voltagegated channels. This not only allows backpropagation of somatic action potentials, but can also lead to initiation of dendritic spikes [63]. A number of different neuronal types can generate dendritic spikes, ranging from cerebellar Purkinje cells, hippocampal and neocortical pyramidal neurons to interneurons. Dendritic spikes can be mediated by different voltage-gated conductances depending on the dendritic compartment and cell type. In pyramidal neurons at proximal locations, dendritic spikes are mediated largely by $\mathrm{Na}^{+}$channels, whereas distal dendritic spikes generally involve activation of voltagegated $\mathrm{Ca}^{2+}$ channels $[15,17,41,45,64]$. Local synaptic stimulation can also evoke NMDA spikes via activation of NMDA-type glutamate receptors [65]. $\mathrm{Ca}^{2+}$ or $\mathrm{Na}^{+}$spikes can also be induced by correlated synaptic inputs [66-68] or bursts of backpropagating action potentials above a critical frequency [41,42] (Figure la,b). Dendritic spikes are usually much slower than axonal action potentials and can occur either in isolation from the soma (local spikes) or induce bursts of axonal action potentials $[61,64,66]$. In addition, pairing distal synaptic inputs with single axonal action potentials at appropriate time intervals can lead to generation of dendritic $\mathrm{Ca}^{2+}$ spikes and subsequent burst firing at the soma, a process termed BAC-firing [61] (Figure Ic-e). The timing requirements for BAC-firing (Figure le) closely match those for STDP at distal synapses (see Figure 4 in main text). Functionally, BAC-firing has been suggested to bind bottom-up sensory information with top-down associative inputs. 
(a)

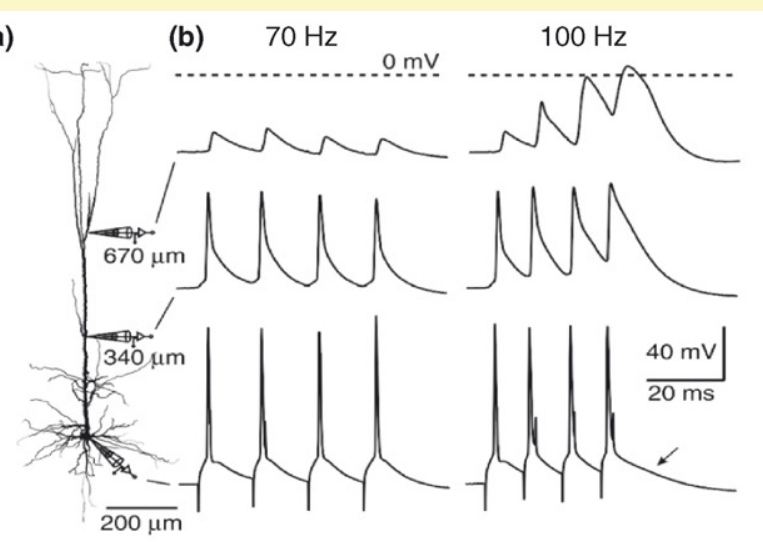

(c)

(d)

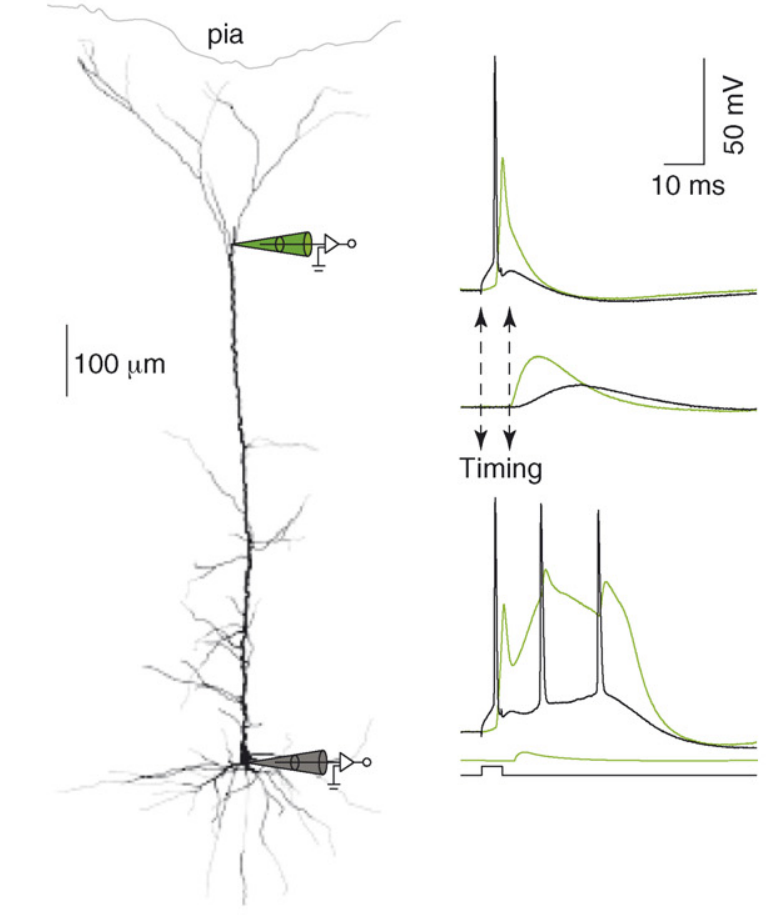

(e)

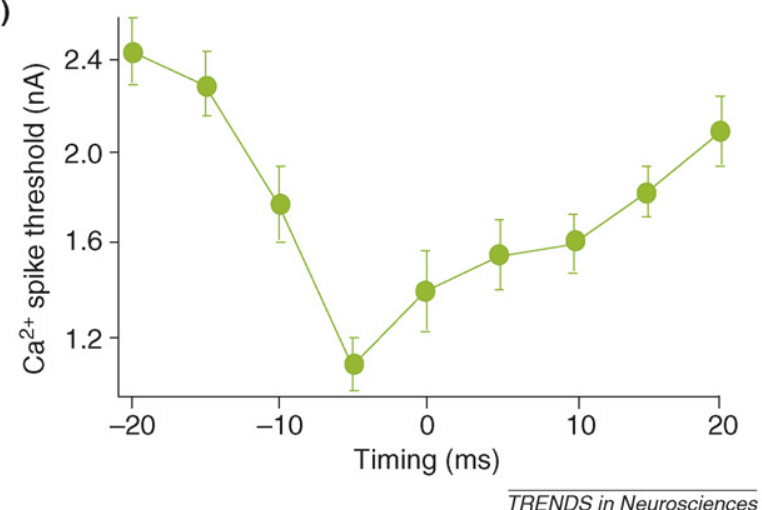

Figure I. Dendritic spikes. (a,b) Action potential bursts beyond a critical frequency generate dendritic $\mathrm{Ca}^{2+}$ spikes. (a) Schematic of the recording situation. (b) Action potentials evoked by brief somatic current injections at the soma of the layer 5 pyramidal neuron and recorded at the indicated locations during low-frequency (left, $70 \mathrm{~Hz}$ ) and high-frequency (right, $100 \mathrm{~Hz}$ ) action potential bursts. Generation of distal dendritic $\mathrm{Ca}^{2+}$ spikes is associated with an increase in the afterdepolarization following the action potential burst (arrow). $(a, b)$ Adapted with permission from [41]. (c,d) Pairing of a distal EPSP (via dendritic current injection) with an axonal action potential at the appropriate timing leads to generation of a dendritic $\mathrm{Ca}^{2+}$ spike and burst firing at the soma (BAC-firing). (c) Schematic of the recording situation. (d) Examples of single during STDP induction. Some forms of STDP might, therefore, be better defined as dendritic-spike-dependent plasticity (or DSDP), as they depend more on the timing of the synaptic input relative to the postsynaptic dendritic spike than on the timing of axonal action potentials. The slower rise time and long duration of dendritic spikes will also confer distinct learning rules to DSDP that will differ from classical STDP [15,44]. Furthermore, the extent and duration of depolarization associated with dendritic spikes differs among dendritic compartments, adding a new level of complexity in which the sign and magnitude of plasticity will depend on dendritic location, as discussed below. Finally, as dendritic spikes can be controlled locally via dendritic $\mathrm{K}^{+}[50]$ or $\mathrm{I}_{\mathrm{h}}$ channels [51,52], as well as GABAergic inhibition [53], the activity state of the surrounding excitatory and inhibitory network will play a crucial role in DSDP induction.

\section{Dendritic mechanisms controlling STDP II: role of synapse location}

Most cortical neurons have extensive dendritic trees, which receive the majority of synaptic contacts. In many instances, specific pathways target different dendritic regions. For example, Schaffer collateral inputs to hippocampal CA1 pyramidal neurons contact the proximal dendrites in the stratum radiatum, whereas inputs from the entorhinal cortex impinge onto distal dendrites in the stratum lacunosum moleculare [54]. In the cortex, layer 5 pyramidal neurons receive bottom-up information from the thalamus primarily via intracolumnar connections from layer $2 / 3$ neurons onto apical and oblique dendrites [28,55], whereas input from neighbouring layer 5 neurons occurs mainly onto basal dendrites [56]. Top-down information from higher cortical areas is transmitted through fibres in layer 1 , which project onto distal apical tuft dendrites of pyramidal neurons $[57,58]$. Together, these observations suggest that synapses onto different dendritic regions convey different kinds of information, which might be integrated in different ways. Consistent with this idea proximal synapses operate mainly by direct depolarization of the axosomatic compartment, whereas integration of distal synaptic inputs is thought to occur through generation of dendritic spikes (and other non-linear processes; Box 1) [59].

Several recent studies have emphasized the importance of dendritic synapse location for STDP induction in cortical pyramidal neurons $[28,38,60]$. The first study of locationdependent STDP showed that the time window for induction of LTD in layer 2/3 pyramidal neurons is broader for distal inputs [60] (Figure 3a,b). The underlying mechanism was found to be greater $\mathrm{Ca}^{2+}$-dependent suppression of NMDA receptors in the distal dendrite. Two recent papers

action potentials (top) or EPSPs (middle) recorded at the soma (black) or dendritic recording location (green) on their own or paired at the appropriate timing leading to BAC-firing (bottom). Dashed lines indicate time interval between somatic action potential and dendritic EPSP. Adapted with permission from [61]. (e) BAC-firing depends on the precise timing of distal EPSPs and postsynaptic action potentials. The graph shows that the minimal EPSP amplitude required to generate BAC-firing is smallest when the distal EPSP occurs just after the postsynaptic action potential (defined as negative timing). Adapted with permission from [61]. 


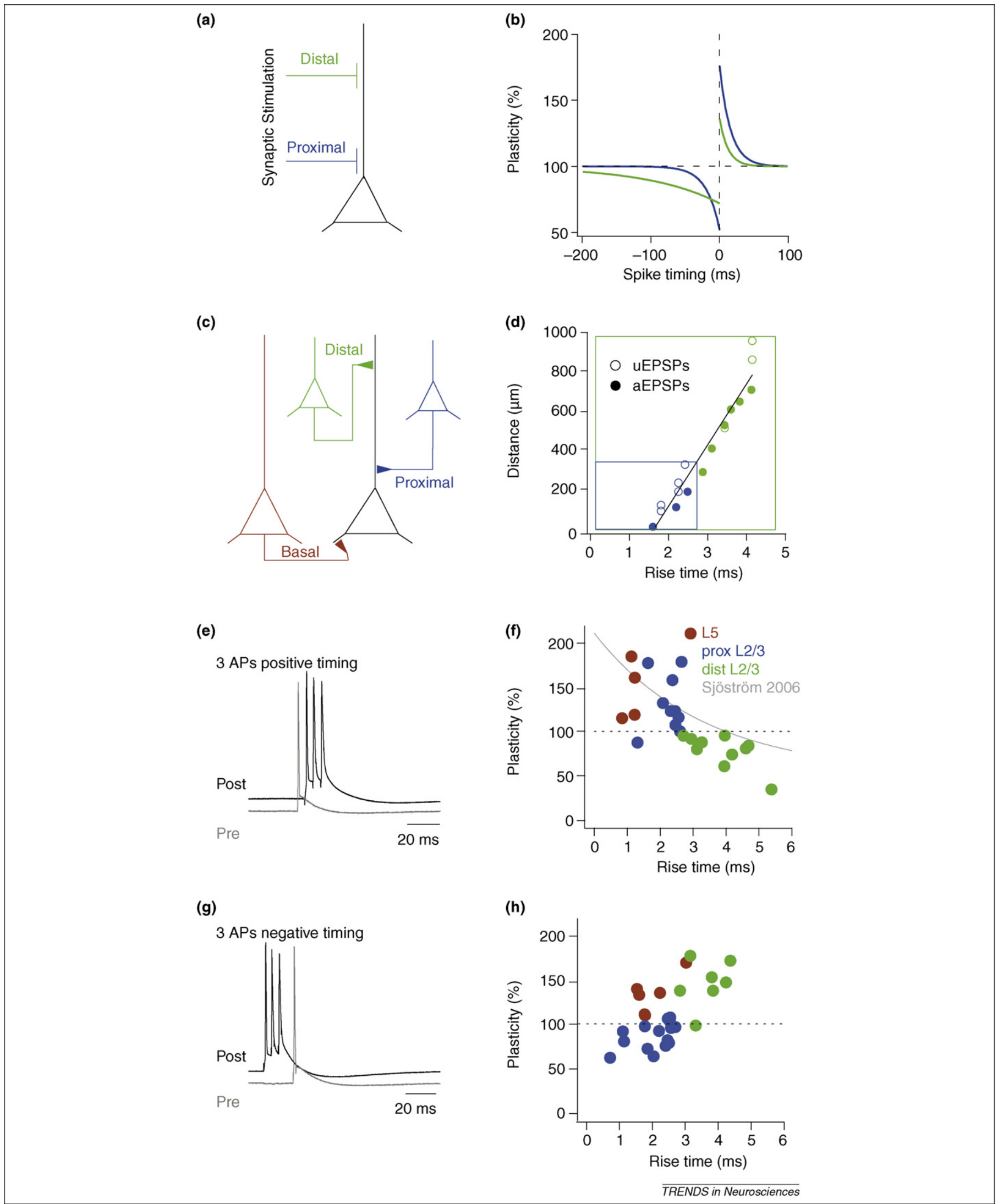

Figure 3. STDP learning rules depend on dendritic synapse location. (a,b) Effect of dendritic synapse location on STDP induction in cortical layer $2 / 3$ pyramidal neurons. (a) Schematic of the experimental arrangement during proximal (blue; $<50 \mu \mathrm{m}$ ) and distal (green; $>100 \mu \mathrm{m}$ ) synaptic stimulation. (b) STDP learning rules for proximal (blue) and distal (green) synapses. Positive spike timing refers to when the synaptic input (EPSP) is evoked before the postsynaptic action potential. Note that the time window for induction of LTD is significantly broader for distal inputs. (a,b) Adapted with permission from [60]. (c) Input pathways onto cortical layer 5 pyramidal neurons studied in the

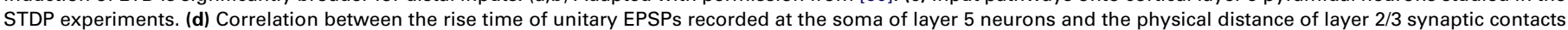
from the soma (open circles). A very similar relationship is observed for artificial EPSPs generated by current injection at known distances along the apical dendritic trunk

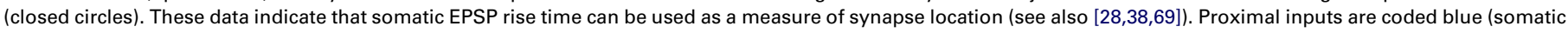


(a)

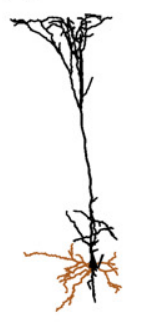

Basal inputs

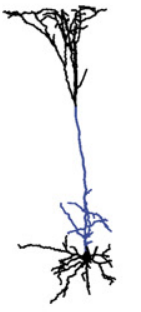

Proximal

apical inputs

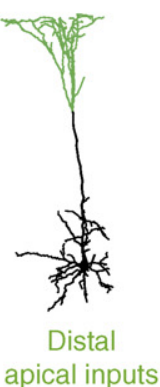

(b)

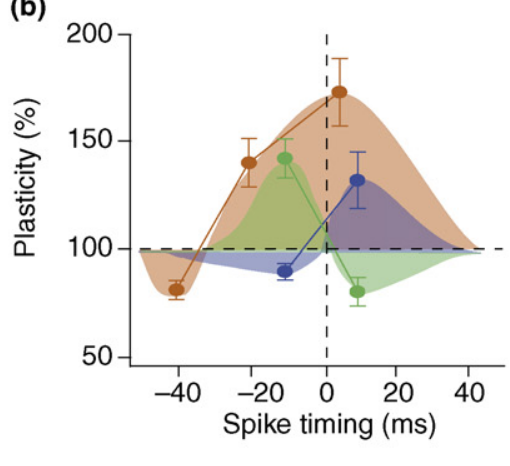

TRENDS in Neurosciences

Figure 4. Pathway-specific STDP timing rules. Dendritic mechanisms can produce distinct learning rules in different dendritic domains of the same neuron. (a) The morphology of a neocortical layer 5 pyramidal neuron with colour-coded input dendritic domains. (b) Hypothetical STDP timing curves for synaptic inputs onto basal dendrites (brown), proximal apical (blue) or distal apical (green) dendrites of cortical layer 5 pyramidal neurons. Data points show mean \pm SEM. Adapted with permission from [15] and [28].

have examined the distance-dependence of STDP in neocortical layer 5 pyramidal neurons. In agreement with previous work, Sjöström and Häusser [38] found that pairing trains of EPSPs and action potentials at positive times leads to LTP at proximal inputs. By contrast, the same paradigm induced LTD at inputs to the distal apical dendrite (Figure 3e,f). Distal LTD was induced even in the absence of postsynaptic firing, but could be converted to LTP by boosting action potential backpropagation after dendritic current injection [38]. Complementary results showed that in layer 5 neurons pairing unitary layer $2 / 3$ inputs with action potential bursts at positive times leads to LTP at proximal synapses and LTD at distal synapses [28] (Figure 3e,f). By contrast, negative pairings had the opposite effect, inducing LTD at proximal inputs and LTP of distal connections [28] (Figure 3g,h).

Thus, whereas proximal inputs from layer $2 / 3$ neurons follow the classical STDP rule with LTP induction for synaptic inputs preceding postsynaptic action potentials and LTD induction for inputs that follow postsynaptic activity [28,38,60], synaptic inputs onto distal parts of the apical dendrite of layer 5 pyramidal neurons can display an opposite learning rule [28,38] (Figure 4). Interestingly, the timing requirement for STDP induction at synapses on distal apical dendrites of layer 5 neurons closely matches that for induction of burst firing by pairing single backpropagating action potentials with distal EPSPs (BAC-firing [61]; Box 1), and both require dendritic spike initiation and negative timing of distal synaptic input relative to the onset of the postsynaptic action potential. By contrast, inputs from neighbouring layer 5 pyramidal neurons, occurring mainly onto basal dendrites, can show different learning rules, with LTP induction at both positive and negative timing intervals during action potential bursts [15] (Figure 4) and NMDA spikes [44].
The emerging picture from these studies is that synapse location has a profound impact on learning rules during STDP. Whereas proximal inputs tend to follow classical STDP rules, distal synapses express novel timing requirements and generally require either dendritic spikes $[15,28,44]$ or dendritic depolarization [38] for LTP induction. These observations parallel the different roles proximal and distal inputs are thought to play in synaptic integration [59].

\section{Conclusions}

Hebb's postulate states that the synaptic connection between two neurons will be strengthened if the two neurons are activated together [4]. Although not proposed by Hebb, there is now also experimental evidence for the converse situation whereby the synaptic connection between two neurons is weakened when the activity of two neurons is uncorrelated. Together these findings form the basis of classical STDP. In this review, we propose an expansion of this model to accommodate recent findings that highlight the influence of dendritic mechanisms in STDP induction. It is now well established that dendrites compartmentalize inputs, increasing the computational power of neurons [62]. In addition, active dendritic properties, such as dendritic spikes, are crucial determinants of synaptic integration. As we review here, there is now emerging evidence that at many cortical synapses dendritic spikes are also required for induction of synaptic plasticity $[15,28,43,44,46]$. This DSDP is in essence an extension of Hebb's postulate that takes into account the importance of dendritic spikes for synaptic integration, particularly at distal synapses. We note that under many conditions dendritic spikes forward propagate to elicit axonal action potentials, so that the link between input and output essential to Hebb's original postulate will often be maintained during DSDP. By contrast, non-Hebbian

rise time $<2.7 \mathrm{~ms}$ ) and distal inputs coded green (somatic rise time $>2.7 \mathrm{~ms}$ ). (c,d) Adapted with permission from [28]. (e-h) Switch of STDP learning rules for distal synaptic inputs. (e) STDP induction paradigm with positive spike timing (refers to when the presynaptic action potential precedes the postsynaptic burst). (f) Induced plasticity for layer 5 to layer 5 inputs (L5, brown), proximal (blue) and distal (green) inputs from layer $2 / 3$ to layer 5 pyramidal neurons plotted versus somatic EPSP for positive timing. Grey curve shows fit to data from [38]. Positive pairing induces LTP at proximal connections and LTD at distal inputs. (g,h) Negative pairing causes the opposite outcome, revealing a switch in STDP learning rules. (e-h) Adapted with permission from [15] and [28]. 
forms of DSDP will occur if dendritic spikes fail to generate axonal action potentials $[43,44,46]$.

In addition to the role of dendritic spikes in plasticity induction, recent data also indicate that the learning rules for STDP depend on synapse location $[28,38,44,60]$. Because most synaptic input is made onto the dendrites, it is the dendritic (not somatic) depolarization that is important for unblocking of synaptic NMDA receptors. It, therefore, comes as no surprise that STDP timing rules depend on synapse location given that the timing, magnitude and duration of backpropagating action potentials and/or dendritic spikes is location dependent. The emerging hypothesis from these studies is that during STDP different dendritic compartments have the capacity to select and maintain inputs conveying different information based on local, rather than global, learning rules.

\section{Acknowledgments}

We would like to thank Fritjof Helmchen, Narimane Benhassine-Hübner and Wiebke Schweer for their comments on the manuscript.

\section{References}

1 Hofer, S.B. et al. (2006) Lifelong learning: ocular dominance plasticity in mouse visual cortex. Curr. Opin. Neurobiol. 16, 451-459

2 Bliss, T.V. and Lømo, T. (1973) Long-lasting potentiation of synaptic transmission in the dentate area of the anaesthetized rabbit following stimulation of the perforant path. J. Physiol. 232, 331-356

3 Lynch, G.S. et al. (1977) Heterosynaptic depression: a postsynaptic correlate of long-term potentiation. Nature 266, 737-739

4 Hebb, D.O. (1949) The Organization of Behavior. Wiley

5 Mayer, M.L. et al. (1984) Voltage-dependent block by $\mathrm{Mg}^{2+}$ of NMDA responses in spinal cord neurones. Nature 309, 261-263

6 Nowak, L. et al. (1984) Magnesium gates glutamate-activated channels in mouse central neurones. Nature 307, 462-465

7 Bliss, T.V. and Collingridge, G.L. (1993) A synaptic model of memory: long-term potentiation in the hippocampus. Nature 361, 31-39

8 Abbott, L.F. and Nelson, S.B. (2000) Synaptic plasticity: taming the beast. Nat. Neurosci. 3, 1178-1183

$9 \mathrm{Bi}, \mathrm{G}-\mathrm{Q}$. and Poo, M-M. (1998) Synaptic modifications in cultured hippocampal neurons: dependence on spike timing, synaptic strength, and postsynaptic cell type. J. Neurosci. 18, 10464-10472

10 Birtoli, B. and Ulrich, D. (2004) Firing mode-dependent synaptic plasticity in rat neocortical pyramidal neurons. J. Neurosci. 24, 4935-4940

11 Nevian, T. and Sakmann, B. (2006) Spine $\mathrm{Ca}^{2+}$ signaling in spiketiming-dependent plasticity. J. Neurosci. 26, 11001-11013

12 Safo, P.K. and Regehr, W.G. (2005) Endocannabinoids control the induction of cerebellar LTD. Neuron 48, 647-659

13 Sjöström, P.J. et al. (2003) Neocortical LTD via coincident activation of presynaptic NMDA and cannabinoid receptors. Neuron 39, 641-654

14 Egger, V. et al. (1999) Coincidence detection and changes of synaptic efficacy in spiny stellate neurons in rat barrel cortex. Nat. Neurosci. 2, 1098-1105

15 Kampa, B.M. et al. (2006) Requirement of dendritic calcium spikes for induction of spike-timing-dependent synaptic plasticity. J. Physiol. $574,283-290$

16 Magee, J.C. and Johnston, D. (1997) A synaptically controlled, associative signal for Hebbian plasticity in hippocampal neurons. Science 275, 209-213

17 Stuart, G. et al. (1997) Action potential initiation and propagation in rat neocortical pyramidal neurons. J. Physiol. 505, 617-632

18 Palmer, L.M. and Stuart, G.J. (2006) Site of action potential initiation in layer 5 pyramidal neurons. J. Neurosci. 26, 1854-1863

19 Stuart, G. et al. (1997) Action potential initiation and backpropagation in neurons of the mammalian CNS. Trends Neurosci. 20, 125-131

20 Kampa, B.M. et al. (2004) Kinetics of $\mathrm{Mg}^{2+}$ unblock of NMDA receptors: implications for spike-timing dependent synaptic plasticity. J. Physiol. $556,337-345$

21 Vargas-Caballero, M. and Robinson, H.P. (2003) A slow fraction of $\mathrm{Mg}^{2+}$ unblock of NMDA receptors limits their contribution to spike generation in cortical pyramidal neurons. J. Neurophysiol. 89, 27782783

22 Debanne, D. et al. (1998) Long-term synaptic plasticity between pairs of individual CA3 pyramidal cells in rat hippocampal slice cultures. J. Physiol. 507, 237-247

23 Koester, H.J. and Sakmann, B. (1998) Calcium dynamics in single spines during coincident pre- and postsynaptic activity depend on relative timing of back-propagating action potentials and subthreshold excitatory postsynaptic potentials. Proc. Natl. Acad. Sci. U. S. A. 95, 9596-9601

24 Nevian, T. and Sakmann, B. (2004) Single spine $\mathrm{Ca}^{2+}$ signals evoked by coincident EPSPs and backpropagating action potentials in spiny stellate cells of layer 4 in the juvenile rat somatosensory barrel cortex. J. Neurosci. 24, 1689-1699

25 Dan, Y. and Poo, M.M. (2004) Spike timing-dependent plasticity of neural circuits. Neuron 44, 23-30

26 Larkman, A.U. (1991) Dendritic morphology of pyramidal neurones of the visual cortex of the rat: III. Spine distributions. J. Comp. Neurol. 306, 332-343

27 Stuart, G.J. and Häusser, M. (2001) Dendritic coincidence detection of EPSPs and action potentials. Nat. Neurosci. 4, 63-71

28 Letzkus, J.J. et al. (2006) Learning rules for spike timing-dependent plasticity depend on dendritic synapse location. J. Neurosci. 26, 10420 10429

29 Markram, H. et al. (1997) Regulation of synaptic efficacy by coincidence of postsynaptic APs and EPSPs. Science 275, 213-215

30 Pike, F.G. et al. (1999) Postsynaptic bursting is essential for 'Hebbian' induction of associative long-term potentiation at excitatory synapses in rat hippocampus. J. Physiol. 518, 571-576

31 Sjöström, P.J. et al. (2001) Rate, timing, and cooperativity jointly determine cortical synaptic plasticity. Neuron 32, 1149-1164

32 Froemke, R.C. and Dan, Y. (2002) Spike-timing-dependent synaptic modification induced by natural spike trains. Nature $416,433-438$

33 Feldman, D.E. (2000) Timing-based LTP and LTD at vertical inputs to layer II/III pyramidal cells in rat barrel cortex. Neuron $27,45-56$

34 Gähwiler, B.H. et al. (1997) Organotypic slice cultures: a technique has come of age. Trends Neurosci. 20, 471-477

35 Lee, A.K. et al. (2006) Whole-cell recordings in freely moving rats. Neuron 51, 399-407

36 Manns, I.D. et al. (2004) Sub- and suprathreshold receptive field properties of pyramidal neurones in layers $5 \mathrm{~A}$ and $5 \mathrm{~B}$ of rat somatosensory barrel cortex. J. Physiol. 556, 601-622

37 Margrie, T.W.et al. (2002) In vivo, low-resistance, whole-cell recordings from neurons in the anaesthetized and awake mammalian brain Pflugers Arch. 444, 491-498

38 Sjöström, P.J. and Häusser, M. (2006) A cooperative switch determines the sign of synaptic plasticity in distal dendrites of neocortical pyramidal neurons. Neuron 51, 227-238

39 Lisman, J.E. (1997) Bursts as a unit of neural information: making unreliable synapses reliable. Trends Neurosci. 20, 38-43

40 Kampa, B.M. and Stuart, G.J. (2006) Calcium spikes in basal dendrites of layer 5 pyramidal neurons during action potential bursts. J. Neurosci. 26, 7424-7432

41 Larkum, M.E. et al. (1999) Calcium electrogenesis in distal apical dendrites of layer 5 pyramidal cells at a critical frequency of backpropagating action potentials. Proc. Natl. Acad. Sci. U. S. A. 96, 1460014604

42 Williams, S.R. and Stuart, G.J. (1999) Mechanisms and consequences of action potential burst firing in rat neocortical pyramidal neurons. J. Physiol. 521, 467-482

43 Golding, N.L. et al. (2002) Dendritic spikes as a mechanism for cooperative long-term potentiation. Nature 418, 326-331

44 Gordon, U. et al. (2006) Plasticity compartments in basal dendrites of neocortical pyramidal neurons. J. Neurosci. 26, 12717-12726

45 Nevian, T. et al. (2007) Properties of basal dendrites of layer 5 pyramidal neurons: a direct patch-clamp recording study. Nat. Neurosci. 10, 206-214

46 Holthoff, K. et al. (2004) Single-shock LTD by local dendritic spikes in pyramidal neurons of mouse visual cortex. J. Physiol. $560,27-36$

47 Goldberg, J. et al. (2002) A problem with Hebb and local spikes. Trends Neurosci. 25, 433-435 
48 Jarsky, T. et al. (2005) Conditional dendritic spike propagation following distal synaptic activation of hippocampal CA1 pyramidal neurons. Nat. Neurosci. 8, 1667-1676

49 Humeau, Y. and Lüthi, A. (2007) Dendritic calcium spikes induce bi-directional synaptic plasticity in the lateral amygdala. Neuropharmacology 52, 234-243

50 Hoffman, D.A. et al. (1997) $\mathrm{K}^{+}$channel regulation of signal propagation in dendrites of hippocampal pyramidal neurons. Nature 387, 869-875

51 Berger, T. et al. (2003) Hyperpolarization-activated current $\mathrm{I}_{\mathrm{h}}$ disconnects somatic and dendritic spike initiation zones in layer $\mathrm{V}$ pyramidal neurons. J. Neurophysiol. 90, 2428-2437

52 Poolos, N.P. et al. (2002) Pharmacological upregulation of h-channels reduces the excitability of pyramidal neuron dendrites. Nat. Neurosci. $5,767-774$

53 Perez-Garci, E. et al. (2006) The $\mathrm{GABA}_{\mathrm{B} 1 \mathrm{~b}}$ isoform mediates longlasting inhibition of dendritic $\mathrm{Ca}^{2+}$ spikes in layer 5 somatosensory pyramidal neurons. Neuron 50, 603-616

54 Witter, M.P. et al. (1989) Functional organization of the extrinsic and intrinsic circuitry of the parahippocampal region. Prog. Neurobiol. 33, 161-253

55 Reyes, A. and Sakmann, B. (1999) Developmental switch in the shortterm modification of unitary EPSPs evoked in layer $2 / 3$ and layer 5 pyramidal neurons of rat neocortex. J. Neurosci. 19, 3827-3835

56 Markram, H. et al. (1997) Physiology and anatomy of synaptic connections between thick tufted pyramidal neurones in the developing rat neocortex. J. Physiol. 500, 409-440

57 Coogan, T.A. and Burkhalter, A. (1990) Conserved patterns of corticocortical connections define areal hierarchy in rat visual cortex. Exp. Brain Res. 80, 49-53
58 Cauller, L.J. et al. (1998) Backward cortical projections to primary somatosensory cortex in rats extend long horizontal axons in layer I. J. Comp. Neurol. 390, 297-310

59 Williams, S.R. and Stuart, G.J. (2003) Role of dendritic synapse location in the control of action potential output. Trends Neurosci. $26,147-154$

60 Froemke, R.C. et al. (2005) Spike-timing-dependent synaptic plasticity depends on dendritic location. Nature 434, 221-225

61 Larkum, M.E. et al. (1999) A new cellular mechanism for coupling inputs arriving at different cortical layers. Nature 398, 338-341

62 Häusser, M. and Mel, B. (2003) Dendrites: bug or feature? Curr. Opin. Neurobiol. 13, 372-383

63 Gulledge, A.T. et al. (2005) Synaptic integration in dendritic trees. J. Neurobiol. 64, 75-90

64 Schiller, J. et al. (1997) Calcium action potentials restricted to distal apical dendrites of rat neocortical pyramidal neurons. J. Physiol. 505, 605-616

65 Schiller, J. et al. (2000) NMDA spikes in basal dendrites of cortical pyramidal neurons. Nature 404, 285-289

66 Williams, S.R. and Stuart, G.J. (2002) Dependence of EPSP efficacy on synapse location in neocortical pyramidal neurons. Science 295, $1907-1910$

67 Gasparini, S. et al. (2004) On the initiation and propagation of dendritic spikes in CA1 pyramidal neurons. J. Neurosci. 24, 11046-11056

68 Losonczy, A. and Magee, J.C. (2006) Integrative properties of radial oblique dendrites in hippocampal CA1 pyramidal neurons. Neuron 50 , 291-307

69 Jack, J.J. et al. (1971) The time course of minimal excitory postsynaptic potentials evoked in spinal motoneurones by group Ia afferent fibres. J. Physiol. 215, 353-380

\section{Elsevier joins major health information initiative}

Elsevier has joined with scientific publishers and leading voluntary health organizations to create patientINFORM, a groundbreaking initiative to help patients and caregivers close a crucial information gap. patientINFORM is a free online service dedicated to disseminating medical research.

Elsevier provides voluntary health organizations with increased online access to our peer-reviewed

biomedical journals immediately upon publication, together with content from back issues. The voluntary health organizations integrate the information into materials for patients and link to the full text of selected research articles on their websites.

patientINFORM has been created to enable patients seeking the latest information about treatment options online access to the most up-to-date, reliable research available for specific diseases.

\section{For more information, visit www.patientinform.org}

